\title{
An mRNA-based vaccine strategy against Zika
}

\author{
Cell Research (2017) 27:1077-1078. doi:10.1038/cr.2017.53; published online 11 April 2017
}

\begin{abstract}
Infections with Zika virus are strongly associated with complications such as congenital microcephaly and can trigger Guillain-Barré syndrome in humans, highlighting the urgent need for a safe, efficacious vaccine as a preventative countermeasure. In a recent paper published in Cell, Richner et al. generated a lipid nanoparticle encapsulated modified mRNA vaccine encoding the prM and $E$ genes from Zika virus, which showed protection and sterilizing immunity in immunocompetent mice.
\end{abstract}

Zika virus (ZIKV) was first discovered by chance from a febrile sentinel macaque during a Yellow fever virus study in Uganda in 1947. For decades afterwards ZIKV was an obscure and neglected mosquito-borne pathogen, and infections have only been documented in Africa and parts of Southeast Asia. Most human cases are typically asymptomatic, and when illness is present it is mild in nature, including fever, conjunctivitis, myalgia, joint pain and maculopapular rash lasting for at most seven days. Carried by Aedes mosquitoes, ZIKV migrated through Micronesia and eventually arrived at Brazil in 2015, where an outbreak of ZIKV fever caused a widespread epidemic across South America, Central America and the Caribbean, reaching as far as southern USA. Many countries that are not geographically close to the Americas also saw imported cases of ZIKV infections from the returning travellers [1]. During the outbreak, it was discovered that ZIKV is not only spread through mosquito bites, but can also be transmitted from male to male, female to male and male to female via sexual contact. Ad- ditionally, ZIKV RNA could be detected in the semen [2] and vaginal secretions [3] of humans at least 3 months after symptom onset. New manifestations of ZIKV fever, including orchitis and potentially male infertility in mice [4], have also been reported. Therefore, the long-term sequelae from ZIKV infections will likely remain a public health concern even after the outbreak is over.

The urgency of the outbreak has spurred the rapid preclinical development and evaluation of several vaccine candidates in the mouse and nonhuman primate (NHP) models, with the aim of licensure as a clinical preventative measure against ZIKV infections. These vaccines typically include the ZIKV matrix (M), pre-matrix (prM) and/or envelope (E) proteins. Currently, the following candidates have been reported: DNA vaccines encoding M-E or prM-E in mice and NHPs [5], adenovirus-vectored vaccine expressing $M-E$ in rhesus macaques [6], and inactivated ZIKV with the alum adjuvant in mice and NHPs [7]. The development of medical countermeasures against ZIKV infections is challenging due to its similarities to the related Dengue virus (DENV). DENV infections are well known for antibody-dependent enhancement (ADE), in which preexisting, cross-reactive antibodies from a prior DENV infection may enhance subsequent infection with a heterologous DENV serotype, turning mild clinical symptoms into a life-threatening disease (Dengue haemorrhagic fever or Dengue shock syndrome). There are concerns that cross-reactive antibodies from DENV can also drive ADE with a subsequent ZIKV infection [8]. A
ZIKV-specific vaccine without the possibility of cross-reactivity to DENV is also desirable.

In a recent paper published in Cell, Richner et al. [9] engineered and tested a modified mRNA-based vaccine against ZIKV in mice. The mRNA was chemically synthesized to contain a type 1 cap as well as $5^{\prime}$ and $3^{\prime}$ untranslated regions (with poly-A tail) to maintain intracellular stability and increase translation efficiency, the signal sequence from human $\operatorname{IgE}$ (IgEsig) for gene expression, and the full-length prM and E genes from an Asian ZIKV strain (IgEsigprM-E). IgEsig-prM-E was then packaged into lipid nanoparticles (LNP) using a modified technique derived from the delivery of siRNA (IgEsig-prM-E LNP), and assessed for vaccine efficacy in immunocompromised AG129 mice as well as immunocompetent $\mathrm{C} 57 \mathrm{BL} / 6$ mice. The results showed that animals that were administered $10 \mu \mathrm{g}$ of IgEsigprM-E LNP via the intramuscular route, followed by an identical boost on day 21 after immunization had high levels of serum neutralizing antibodies (EC90 $=1 / 1000)$ before challenge. These animals showed $100 \%$ survival without weight loss when challenged with ZIKV 42 days after vaccination.

Based on these results, the authors then explored methods to minimize the possibility of ADE. In addition to replacing IgEsig with the corresponding sequence from Japanese encephalitis virus (JEVsig) to increase expression levels, the authors tested a modified mRNA vaccine in which the $\mathrm{E}$ gene has been mutated such that it lacks the immunodominant fusion loop (FL) epitopes in domain II, thereby 
eliminating the ability of the vaccine to generate cross-reactive antibodies. The immunogenicity of this vaccine candidate (JEVsig-prM-E FL LNP) was tested alongside a control vaccine with wild-type (WT) E (JEVsig-prM-E WT $\mathrm{LNP}$ ) in vivo in $\mathrm{BALB} / \mathrm{c}$ mice with the same regimens as described above, and the FL-vaccinated mice showed 10-fold lower antibody responses compared to WT-vaccinated mice. Sterile immunity was observed in the WT-vaccinated mice when challenged 13 weeks after vaccination, but not observed in FLvaccinated mice, as evidenced by the presence of ZIKV RNA at low levels in the sera, spleen and brain from these animals. Passive serum transfer from mice immunized with JEVsig-prM-E WT LNP to naive AG129 mice resulted in $\mathrm{ADE}$ and $100 \%$ mortality when subsequently challenged with DENV-2, whereas ADE was not readily observable in AG129 mice passively given sera from JEVsig-prM-E FL LNP, with a $10 \%-20 \%$ death rate.

The main advantage of mRNA-based vaccines is the ability to be modified at will to enhance immunogenicity or eliminate undesired side effects, such as the induction of cross-reactive antibodies leading to ADE. In their study, the authors show that cross-reactivity can be minimized from ZIKV vaccines, but at the cost of immunogenicity. While other mutations that abolish the FL epitopes are possible and may result in improved neutralizing antibody responses, based on current evidence it is likely that multiple booster injections will be needed to confer sterilizing immunity without cross-reactivity. Furthermore, ZIKV vaccines should also be tested for immunogenicity and efficacy in the presence of pre-existing immunity with DENV, as cross-reactive antibodies from DENV may negatively impact protective immune responses to ZIKV [10]. To bring a vaccine to market, a statistically robust immune correlate of protection must be established to reliably predict vaccinemediated protection without the need for challenge with ZIKV. Neutralizing antibodies may be a good choice in this regard, as they are shown to have dose-dependent, therapeutic properties against ZIKV infections [11]. The safety of mRNA-based vaccines in humans will also need to be characterized in clinical trials. Therefore, it is unlikely that a licensed ZIKV vaccine is on the immediate horizon, but the results of this study constitute an important step towards this goal.
Gary Wong ${ }^{1,2}$,

George F Gao ${ }^{1,2}$

${ }^{1}$ CAS Key Laboratory of Pathogenic Microbiology and Immunology, Institute of Microbiology, Chinese Academy of Sciences, Beijing, China; ${ }^{2}$ Shenzhen Key Laboratory of Pathogen and Immunity, State Key Discipline of Infectious Disease, Shenzhen Third People's Hospital, Shenzhen, Guangdong, China

Correspondence: Gary Wong

E-mail: garyckwong@hotmail.com

\section{References}

1 Zhang Y, Chen W, Wong G, et al. Protein Cell 2016; 7:461-464.

2 Mansuy JM, Pasquier C, Daudin M, et al. Lancet Infect Dis 2016; 16:894-895.

3 Murray KO, Gorchakov R, Carlson AR, et al. Emerg Infect Dis 2017; 23:99-101.

4 Ma W, Li S, Ma S, et al. Cell 2016; 167:1511-1524.

5 Dowd KA, Ko SY, Morabito KM, et al. Science 2016; 354:237-240.

6 Abbink P, Larocca RA, De La Barrera RA, et al. Science 2016; 353:1129-1132.

7 Larocca RA, Abbink P, Peron JP, et al. Nature 2016; 536:474-478.

8 Dejnirattisai W, Supasa P, Wongwiwat W, et al. Nat Immunol 2016; 17:1102-1108.

9 Richner JM, Himansu S, Dowd KA, et al. Cell 2017; 168:1114-1125.

10 Priyamvada L, Quicke KM, Hudson WH, et al. Proc Natl Acad Sci USA 2016; 113:78527857.

11 Wang Q, Yang H, Liu X, et al. Sci Transl Med 2016; 8:369ra179. 